\title{
Competition and resource sensitivity in marriage and roommate markets
}

Citation for published version (APA):

Klaus, B. E. (2007). Competition and resource sensitivity in marriage and roommate markets. METEOR, Maastricht University School of Business and Economics. METEOR Research Memorandum No. 046 https://doi.org/10.26481/umamet.2007046

Document status and date:

Published: 01/01/2007

DOI:

10.26481/umamet.2007046

Document Version:

Publisher's PDF, also known as Version of record

\section{Please check the document version of this publication:}

- A submitted manuscript is the version of the article upon submission and before peer-review. There can be important differences between the submitted version and the official published version of record.

People interested in the research are advised to contact the author for the final version of the publication, or visit the DOI to the publisher's website.

- The final author version and the galley proof are versions of the publication after peer review.

- The final published version features the final layout of the paper including the volume, issue and page numbers.

Link to publication

\footnotetext{
General rights rights.

- You may freely distribute the URL identifying the publication in the public portal. please follow below link for the End User Agreement:

www.umlib.nl/taverne-license

Take down policy

If you believe that this document breaches copyright please contact us at:

repository@maastrichtuniversity.nl

providing details and we will investigate your claim.
}

Copyright and moral rights for the publications made accessible in the public portal are retained by the authors and/or other copyright owners and it is a condition of accessing publications that users recognise and abide by the legal requirements associated with these

- Users may download and print one copy of any publication from the public portal for the purpose of private study or research.

- You may not further distribute the material or use it for any profit-making activity or commercial gain

If the publication is distributed under the terms of Article $25 \mathrm{fa}$ of the Dutch Copyright Act, indicated by the "Taverne" license above, 


\section{Bettina Klaus}

Competition and Resource Sensitivity in Marriage and Roommate Markets

$\mathrm{RM} / 07 / 046$

JEL code: C78, D63

\section{METEबrR}

Maastricht research school of Economics of TEchnology and ORganizations

Universiteit Maastricht

Faculty of Economics and Business Administration P.O. Box 616

NL - 6200 MD Maastricht

phone : ++31433883830

fax : ++31433884873 


\title{
Competition and Resource Sensitivity in Marriage and Roommate Markets*
}

\author{
Bettina Klaus ${ }^{\dagger}$
}

November 2007

\begin{abstract}
We consider one-to-one matching markets in which agents can either be matched as pairs or remain single. In these so-called roommate markets agents are consumers and resources at the same time. We investigate two new properties that capture the effect a newcomer has on incumbent agents. Competition sensitivity focuses on the newcomer as additional consumer and requires that some incumbents will suffer if competition is caused by a newcomer. Resource sensitivity focuses on the newcomer as additional resource and requires that this is beneficial for some incumbents. For solvable roommate markets, we provide the first characterizations of the core using either competition or resource sensitivity. On the class of all roommate markets, we obtain two associated impossibility results.
\end{abstract}

JEL classification: C78, D63.

Keywords: Core, Matching, Competition Sensitivity, Resource Sensitivity, Roommate Market.

${ }^{*}$ B. Klaus thanks the Netherlands Organisation for Scientific Research (NWO) for its support under grant VIDI-452-06-013. I also wish to thank Elena Molis and Manabu Toda for their valuable comments and interesting discussions.

${ }^{\dagger}$ Department of Economics, Maastricht University, P.O. Box 616, 6200 MD Maastricht, The Netherlands; e-mail: b.klaus@algec.unimaas.nl 


\section{Introduction}

We consider one-to-one matching markets in which agents can either be matched as pairs or remain single. These markets are known as roommate markets and they include as special cases the well-known marriage markets (Gale and Shapley, 1962; Roth and Sotomayor, 1990). As simple as the roommate model may be, it is of conceptual importance as it lies in the intersection of network and coalition formation models ${ }^{1}$ (for surveys and current research of network and coalition formation see Demange and Wooders, 2004).

Loosely speaking, in these discrete markets the commodities to be traded are the agents themselves. Thus, agents are consumers and resources at the same time. We investigate two new properties that capture the effect a newcomer has on incumbent agents: competition and resource sensitivity. Competition sensitivity focuses on the newcomer as additional consumer and requires that some incumbents will suffer if competition is caused because the newcomer initiates new trades. Resource sensitivity focuses on the newcomer as additional resource and requires that some incumbents will benefit if there are new trades, i.e., the extra resource is consumed.

For marriage markets, both properties are closely related to population monotonicity, a solidarity property that requires that additional agents affect the incumbents in a similar way (either all incumbents are weakly better off or all incumbents are weakly worse off). Because of the polarization of interests that occurs in marriage markets, two specific versions of population monotonicity exist: own-side and other-side population monotonicity (Toda, 2006 , indroduced the first of these specifications). ${ }^{2}$ We show that in marriage markets, essentially own-side population monotonicity implies competition sensitivity (Lemma 1) and other-side population monotonicity implies resource sensitivity (Lemma 2). Our main results are two characterizations of the core by unanimity ${ }^{3}$, Maskin monotonicity ${ }^{4}$, and either competition or resource sensitivity for solvable roommate markets (Theorem 1) and two associated impossibility results on the general domain (Theorem 2).

Theorem 1 presents the first characterizations of the core for solvable roommate markets. One of Toda's (2006, Theorem 3.1) results can be interpreted as a corollary (Corollary 1) of our results. More importantly, Theorem 1 demonstrates that it is not really a solidarity property (population monotonicity) that is at work in Toda's (2006) characterization of the core for marriage markets, but that it is the competition sensitivity aspect that is captured as well. Our results also imply a new characterization of the core for marriage markets (Corollary 2): a solution $\varphi$ satisfies unanimity, Maskin monotonicity, and other-side population monotonicity if and only if it equals the core.

\footnotetext{
${ }^{1}$ In a "roommate network" situation each agent is allowed or able to form only one link and in a "roommate coalition" situation only coalitions of size one or two can be formed.

${ }^{2}$ Own-side population monotonicity: if additional men (women) enter the market, then all incumbent men (women) are weakly worse off.

Other-side population monotonicity: if additional men (women) enter the market, then all incumbent women (men) are weakly better off.

${ }^{3}$ Unanimity: if a unanimously best matching exists, then it is chosen.

${ }^{4}$ Maskin monotonicity: if a matching is chosen in one market, then it is also chosen in a market that results from a Maskin monotonic transformation (which essentially means that the matching improved in the ranking of all agents).
} 


\section{Roommate Markets}

\subsection{The Model}

Gale and Shapley (1962, Example 3) introduced the very simple and appealing roommate markets as follows: "An even number of boys wish to divide up into pairs of roommates." A very common extension of this problem is to allow also for odd numbers of agents and to consider the formation of pairs and singletons (rooms can be occupied either by one or by two agents). In addition, we will extend the problem to variable sets of agents, e.g., because the allocation of dormitory rooms at a university occurs every year for different sets of students.

Let $\mathbb{N}$ be the set of potential agents ${ }^{5}$ and $\mathcal{N}$ be the set of all non-empty finite subsets of $\mathbb{N}$, i.e., $\mathcal{N}=\{N \subseteq \mathbb{N}|\infty>| N \mid>0\}$. For $N \in \mathcal{N}, L(N)$ denotes the set of all linear orders over $N{ }^{6}$ For $i \in N$, we interpret $R_{i} \in L(N)$ as agent $i$ 's preferences over sharing a room with any of the agents in $N \backslash\{i\}$ and having a room for himself; e.g., $R_{i}: j, k, i, l$ means that $i$ would first like to share a room with $j$, then with $k$, and then $i$ would prefer to stay alone rather than sharing the room with $l$. If $j P_{i} i$ then agent $i$ finds agent $j$ acceptable and if $i P_{i} j$ then agent $i$ finds agent $j$ unacceptable. $\mathcal{R}^{N}=\prod_{N} L(N)$ denotes the set of all preference profiles of agents in $N$ (over agents in $N$ ). A roommate market consists of a set of agents $N \in \mathcal{N}$ and their preferences $R \in \mathcal{R}^{N}$ and is denoted by $(N, R)$. A marriage market (Gale and Shapley, $1962)$ is a roommate market $(N, R)$ such that $N$ is the union of two disjoint sets $M$ and $W$, and each agent in $M$ (respectively $W$ ) prefers being single to being matched with any other agent in $M$ (respectively $W$ ).

A matching $\mu$ for roommate market $(N, R)$ is a function $\mu: N \rightarrow N$ of order two, i.e., for all $i \in N, \mu(\mu(i))=i$. Thus, at any matching $\mu$, the set of agents is partitioned into pairs of agents who share a room and singletons (agents who do not share a room). Agent $\mu(i)$ is agent $i$ 's match (if $\mu(i)=i$ then $i$ is matched to himself or single). For marriage markets, a matching never matches two men or two women (the partition consists of man-woman pairs and singletons). For $S \subseteq N$, we denote by $\mu(S)$ the set of agents that are matched to agents in $S$, i.e., $\mu(S)=\left\{i \in N \mid \mu^{-1}(i) \in S\right\}$. We denote the set of matchings for roommate market $(N, R)$ by $\mathcal{M}(N, R)$ (even though this set does not depend on preferences $R$ ). If it is clear which roommate market $(N, R)$ we refer to, matchings are assumed to be elements of $\mathcal{M}(N, R)$. We use the same notation for preferences over agents and matchings: for all agents $i \in N$ and matchings $\mu, \mu^{\prime}, \mu R_{i} \mu^{\prime}$ if and only if $\mu(i) R_{i} \mu^{\prime}(i)$.

A solution $\varphi$ is a correspondence that associates with each roommate market $(N, R)$ a nonempty subset of matchings, i.e., for all $(N, R), \varphi(N, R) \subseteq \mathcal{M}(N, R)$ and $\varphi(N, R) \neq \emptyset$.

\subsection{Basic Properties and the Core}

We first introduce a voluntary participation condition based on the idea that no agent can be forced to share a room.

\footnotetext{
${ }^{5}$ Only Lemma 3, Theorems 1 (a), 2 (a), and Corollary 1 depend on the set of potential agents to be infinite. Propositions 1, 2, Lemmas 1, 2, 4, 5, 6, 7, Theorems 1 (b), 2 (b), and Corollary 2 are also valid for a finite set of potential agents.

${ }^{6} \mathrm{~A}$ linear order over $N$ is a binary relation $\bar{R}$ that satisfies antisymmetry (for all $i, j \in N$, if $i \bar{R} j$ and $j \bar{R} i$, then $i=j$ ), transitivity (for all $i, j, k \in N$, if $i \bar{R} j$ and $j \bar{R} k$, then $i \bar{R} k$ ), and comparability (for all $i, j \in N$, $i \bar{R} j$ or $j \bar{R} i$ ). By $\bar{P}$ we denote the asymmetric part of $\bar{R}$. Hence, given $i, j \in N, i \bar{P} j$ means that $i$ is strictly preferred to $j$; $i \bar{R} j$ means that $i \bar{P} j$ or $i=j$ and that $i$ is weakly preferred to $j$.
} 


\section{Definition 1. Individual Rationality}

A matching $\mu$ is individually rational for roommate market $(N, R)$ if for all $i \in N, \mu(i) R_{i} i$. $I R(N, R)$ denotes the set of all individually rational matchings for roommate market $(N, R)$. A solution $\varphi$ is individually rational if it only assigns individually rational matchings, i.e., for all $(N, R), \varphi(N, R) \subseteq I R(N, R)$.

Next, we introduce the well-known condition of Pareto optimality and the weaker condition of unanimity.

\section{Definition 2. Pareto Optimality}

A matching $\mu$ is Pareto optimal for roommate market $(N, R)$ if there is no other matching $\mu^{\prime} \in \mathcal{M}(N, R)$ such that for all $i \in N, \mu^{\prime} R_{i} \mu$ and for some $j \in N, \mu^{\prime} P_{j} \mu$. PO $(N, R)$ denotes the set of all Pareto optimal matchings for roommate market $(N, R)$. A solution $\varphi$ is Pareto optimal if it only assigns Pareto optimal matchings, i.e., for all $(N, R), \varphi(N, R) \subseteq P O(N, R)$.

\section{Definition 3. Unanimity}

Let $(N, R)$ be a roommate market and $\mu$ be such that for all $i, j \in N, \mu(i) R_{i} j$. Then, $\mu$ is the unanimously best matching for $(N, R)$. A solution $\varphi$ is unanimous if it assigns the unanimously best matching whenever it exists, i.e., for all roommate markets $(N, R)$ with an unanimously best matching $\mu, \varphi(N, R)=\{\mu\}$.

Throughout the paper we could also use a somewhat weaker version of unanimity: a solution $\varphi$ is weakly unanimous if it chooses the unanimously best matching whenever it exists and is complete (no agent is single). We define weak unanimity and show how the relevant proofs (the proofs of Lemmas 3 and 4) should be adjusted in Appendix C. Note that Pareto optimality implies unanimity and that unanimity implies weak unanimity.

The next property requires that two agents who are "mutually best agents" are always matched with each others.

\section{Definition 4. Mutually Best}

Let $(N, R)$ be a roommate market and $i, j \in N$ [possibly $i=j$ ] such that for all $k \in N, i R_{j} k$ and $j R_{i} k$. Then, $i$ and $j$ are mutually best agents for $(N, R)$. A solution $\varphi$ is mutually best if it only assigns matchings at which all mutually best agents are matched, i.e., for all roommate markets $(N, R)$, for all mutually best agents $i$ and $j$, and for all $\mu \in \varphi(N, R), \mu(i)=j$.

Note that mutually best implies unanimity and that Pareto optimality and mutually best are logically unrelated.

Examples of solutions are implied by two of the properties: define $I R$ (respectively $P O$ ) as correspondences that assign to each roommate market the set of individually rational (respectively Pareto optimal) matchings. Another well-known solution is the core.

\section{Definition 5. Core}

A matching is in the (strict or strong) core if no coalition of agents can improve their welfare by rematching among themselves. For roommate market $(N, R)$, core $(N, R)=\{\mu \in \mathcal{M}(N, R) \mid$ there exists no $S \subseteq N$ and no $\mu^{\prime} \in \mathcal{M}(N, R)$ such that $\mu^{\prime}(S)=S$, for all $i \in S, \mu^{\prime}(i) R_{i}$ $\mu(i)$, and for some $\left.j \in S, \mu^{\prime}(j) P_{j} \mu(j)\right\}$.

Next, we define stability for roommate markets. A matching $\mu$ is blocked by a pair $\{i, j\} \subseteq N$ [possibly $i=j$ ] if $j P_{i} \mu(i)$ and $i P_{j} \mu(j)$. If $\{i, j\}$ blocks $\mu$, then $\{i, j\}$ is called a blocking pair for $\mu$. Note that a matching is individually rational if there is no blocking pair $\{i, j\}$ with $i=j$. 


\section{Definition 6. Stability}

A matching $\mu$ is stable for roommate market $(N, R)$ if there is no blocking pair for $\mu$. $S(N, R)$ denotes the set of all stable matchings for roommate market $(N, R)$. A solution $\varphi$ is stable if it only assigns stable matchings, i.e., for all $(N, R), \varphi(N, R) \subseteq S(N, R)$.

Similarly as in other matching models (e.g., marriage markets and college admissions markets), the core equals the set of stable matchings, i.e., for all $(N, R)$, core $(N, R)=S(N, R)$. A roommate market is solvable if stable/core matchings exist. Gale and Shapley (1962) showed that all marriage markets are solvable and gave an example of an unsolvable roommate market (Gale and Shapley, 1962, Example 3).

Finally, we introduce Maskin monotonicity (Maskin, 1999): if a matching is chosen in one roommate market, then it is also chosen in a roommate market that results from a Maskin monotonic transformation, which essentially means that the matching (weakly) improved in the preference ranking of all agents.

Let $(N, R)$ be a roommate market. Then, for any agent $i \in N$ and matching $\mu \in \mathcal{M}(N, R)$, the lower contour set of $R_{i}$ at $\mu$ is $L_{i}\left(R_{i}, \mu\right):=\left\{\mu^{\prime} \in \mathcal{M}(N, R) \mid \mu R_{i} \mu^{\prime}\right\}$. For preference profiles $R, R^{\prime} \in \mathcal{R}^{N}$ and matching $\mu \in \mathcal{M}(N, R), R^{\prime}$ is a Maskin monotonic transformation of $R$ at $\mu$ if for all $i \in N, L_{i}\left(R_{i}, \mu\right) \subseteq L_{i}\left(R_{i}^{\prime}, \mu\right)$.

\section{Definition 7. Maskin Monotonicity}

A solution $\varphi$ is Maskin monotonic if for all roommate markets $(N, R),\left(N, R^{\prime}\right)$, and all $\mu \in$ $\varphi(N, R)$ such that $R^{\prime}$ is a Maskin monotonic transformation of $R$ at $\mu, \mu \in \varphi\left(N, R^{\prime}\right)$.

Maskin monotonicity is one of the key concepts in implementation theory. However, here we focus on Maskin monotonicity as a desirable property in itself.

Proposition 1. On the class of solvable roommate markets, the core satisfies individual rationality, Pareto optimality, unanimity, mutually best, stability, and Maskin monotonicity.

Proof. It is easily checked that the core satisfies individual rationality, Pareto optimality, unanimity, mutually best, and stability. Sönmez (1996, Proposition 1) showed that the core is Maskin monotonic.

\subsection{Variable Population Properties}

The next properties we consider concern population changes. More specifically, consider the change of a roommate market $(N, R)$ when one new agent, a newcomer $n \in \mathbb{N} \backslash N$, shows up. Then, the new set of agents is $N^{\prime}=N \cup\{n\}$ and $\left(N^{\prime}, R^{\prime}\right), R^{\prime} \in \mathcal{R}^{N^{\prime}}$, is an extension of $(N, R)$ if agents in $N$ extend their preferences to include $n$, i.e.,

(i) for all $i \in N^{\prime}, R_{i}^{\prime} \in L\left(N^{\prime}\right)$ and

(ii) for all $j, k, l \in N, j R_{l} k$ if and only if $j R_{l}^{\prime} k$.

Note that $R \in \mathcal{R}^{N}$ is the restriction of $R^{\prime} \in \mathcal{R}^{N^{\prime}}$ to $N$. We also denote the restriction of $R^{\prime}$ to $N$ by $R_{\mid N}^{\prime}$.

Adding an agent $n$ might be a positive or a negative change for any of the incumbents in $N$ because it might mean

a negative change with more competition or

a positive change with more resources. 
Before we capture both effects in two new properties called competition and resource sensitivity, we make a short excursion to the definition of population monotonicity for marriage markets. This property goes back to Thomson (1983), who also presents a survey of population monotonicity in various economic models (Thomson, 1995).

Population Monotonicity: When a change in the population is exogenous, it would be unfair if the agents who were not responsible for this change were treated unequally. Population monotonicity represents this idea of solidarity. However, for marriage markets this would mean that if a newcomer enters (e.g., a man) men and women are all affected in the same way (all weakly better off or all weakly worse off).

This might not be a natural condition for marriage markets because of a certain polarization imbedded in the market: a man might be considered good news for women (more choice), but bad news for men (more competition). Therefore, for marriage markets we can formulate two population monotonicity conditions that take the polarization aspect into account. The first one was introduced by Toda (2006) and we will refer to it as own-side population monotonicity: a solution $\varphi$ is own-side population monotonic if for any marriage market $(M \cup W, R)$, if additional men [women] enter the market such that the new marriage market equals $\left(M^{\prime} \cup W, R^{\prime}\right)\left[\left(M \cup W^{\prime}, R^{\prime}\right)\right]$, then - because of the possible negative effect of the extra competition - all men in $M$ [women in $W$ ] weakly prefer $\varphi(M \cup W, R)$ to $\varphi\left(M^{\prime} \cup W, R^{\prime}\right)$ $\left[\varphi\left(M \cup W^{\prime}, R^{\prime}\right)\right]$.

We formalize a somewhat weaker version of own-side population monotonicity by restricting population changes to one agent at a time. Consistent with Toda's (2006) choice of extending preferences over matchings to sets of matchings, we apply the pessimistic view of comparing sets of matchings. ${ }^{7}$

\section{Definition 8. Own-Side Population Monotonicity for Marriage Markets}

A solution $\varphi$ is own-side population monotonic if the following holds. Let $(N, R)$ be a marriage market and assume that $\left(N^{\prime}, R^{\prime}\right), N^{\prime}=N \cup\{n\}$, is an extension of $(N, R)$ and the newcomer $n$ is a man [woman]. Then, for all $\mu \in \varphi(N, R)$ there exists $\mu^{\prime} \in \varphi\left(N^{\prime}, R^{\prime}\right)$ such that (*) for all men $m \in N, \mu(m) R_{m} \mu^{\prime}(m)$ [for all women $w \in N, \mu(w) R_{w} \mu^{\prime}(w)$ ].

By the strictness of preferences, $(*)$ means that if the newcomer is a man [woman], then every man [woman] who is matched differently is strictly worse off, i.e., for all men $m \in N$, either $\mu(m)=\mu^{\prime}(m)$ or $\mu(m) P_{m} \mu^{\prime}(m)$ [for all women $w \in N$, either $\mu(w)=\mu^{\prime}(w)$ or $\left.\mu(w) P_{w} \mu^{\prime}(w)\right]$. Hence, if a man $m$ [woman $w$ ] has a new mate at $\mu^{\prime}$, then he [she] is worse off. Without specifying whether the newcomer is a man or a woman, own-side population monotonicity implies that if $m, w \in N$ are new mates at $\mu^{\prime}$, then at least one of them is worse off (if the newcomer is a man, then man $m$ is worse off and if the newcomer is a woman, then woman $w$ is worse off). This latter requirement that if two incumbents are newly matched at $\mu^{\prime}$, then one of them suffers from the increased competition by the newcomer and is worse off, can be formulated as a new property, namely competition sensitivity. This property requires that the solution is sensitive to competition, which is a different requirement than the solidarity aspect that own-side population monotonicity reflects.

\footnotetext{
${ }^{7}$ Agents are pessimistic and always assume that the worst matching will be realized, i.e., given two sets of matchings $A$ and $B$, an agent will compare the worst matching in $A$ to the worst matching in $B$. Thus, if agent $i$ weakly prefers $A$ to $B$, then for all $\mu \in A$ there exists $\mu^{\prime} \in B$ such that $\mu R_{i} \mu^{\prime}$.

As already noted by Toda (2006), using an optimistic set comparison, i.e., comparing the best matchings, will not give the same results and using a standard set comparison that compares best to best and worst to worst matchings (see Barberà et al., 2004) will not change the results.
} 


\section{Definition 9. Competition Sensitivity}

A solution $\varphi$ is competition sensitive if the following holds. Let $(N, R)$ be a roommate market and assume that $\left(N^{\prime}, R^{\prime}\right), N^{\prime}=N \cup\{n\}$, is an extension of $(N, R)$. Then, for all $\mu \in \varphi(N, R)$ there exists $\mu^{\prime} \in \varphi\left(N^{\prime}, R^{\prime}\right)$ such that for all $i, j \in N$ [possibly $i=j$ ] that are new mates at $\mu^{\prime}$ at least one is worse off, i.e., if $i, j \in N, \mu(i) \neq j$, and $\mu^{\prime}(i)=j$, then $\mu(i) P_{i} \mu^{\prime}(i)$ or $\mu(j) P_{j} \mu^{\prime}(j)$.

On the class of marriage markets, competition sensitivity is essentially a weaker property than own-side population monotonicity.

\section{Lemma 1. Own-Side Population Monotonicity $\Rightarrow$ Competition Sensitivity}

On the class of marriage markets, individual rationality and own-side population monotonicity imply competition sensitivity.

Proof. Let $\varphi$ be a solution that is individually rational and own-side population monotonic on the class of marriage markets. Let $(N, R)$ be a marriage market and assume that $\left(N^{\prime}, R^{\prime}\right)$, $N^{\prime}=N \cup\{n\}$, is an extension of $(N, R)$. Without loss of generality assume that $n$ is a man. By own-side population monotonicity, for all $\mu \in \varphi(N, R)$ there exists $\mu^{\prime} \in \varphi\left(N^{\prime}, R^{\prime}\right)$ such that for all men $m \in N$, either $\mu(m)=\mu^{\prime}(m)$ or $\mu(m) P_{m} \mu^{\prime}(m)$. Let $i, j \in N, \mu(i) \neq j$, and $\mu^{\prime}(i)=j$. If $i \neq j$, then the pair $\{i, j\}$ consists of one man and one woman. Without loss of generality assume that $i$ is the man and $j$ the woman. Thus, by own-side population monotonicity, $\mu(i) P_{i} \mu^{\prime}(i)$. If $i=j$, then, by individual rationality, $\mu(i) P_{i} \mu^{\prime}(i){ }^{8}$ Hence, $\varphi$ is competition sensitive.

In the following example we demonstrate that for marriage markets, competition sensitivity is indeed a weaker condition than own-side population monotonicity.

Example 1. Assume, without loss of generality, that there exist women $w_{1}, w_{2} \in \mathbb{N}$ and men $m_{1}, m_{2} \in \mathbb{N}$ such that $w_{1}<m_{1}$ and $m_{2}<w_{2}$. Solution $\bar{\varphi}$ uses two important stable matchings that always exist for marriage markets: the man- and the woman-optimal stable matching (obtainable by applying the deferred acceptance algorithm, cf., Gale and Shapley, 1962). We define $\bar{\varphi}$ as follows. For all marriage markets $(N, R)$, if the agent with the largest index is a man, then $\bar{\varphi}$ assigns the man-optimal stable matching and otherwise $\bar{\varphi}$ assigns the woman-optimal stable matching. Solution $\bar{\varphi}$ is individually rational and competition sensitive (see Proposition 2), but it violates own-side population-monotonicity (see Appendix A). $\diamond$

Next we introduce other-side population monotonicity for marriage markets: a solution $\varphi$ is other-side population monotonic if for any marriage market $(M \cup W, R)$, if additional men [women] enter the market such that the new marriage market equals $\left(M^{\prime} \cup W, R^{\prime}\right)[(M \cup$ $\left.\left.W^{\prime}, R^{\prime}\right)\right]$, then - because of the possible positive effect of the extra matching opportunities or resources - all women in $W$ [men in $M]$ weakly prefer $\varphi\left(M^{\prime} \cup W, R^{\prime}\right)\left[\varphi\left(M \cup W^{\prime}, R^{\prime}\right)\right]$ to $\varphi(M \cup W, R)$.

Again, we formalize a somewhat weaker version of other-side population monotonicity by restricting population changes to one agent at a time.

\footnotetext{
${ }^{8}$ If $i$ is a man, this latter implication would also be implied by own-side population monotonicity. However, if $i$ is a woman, this concluding argument cannot be made solely by using own-side population monotonicity.
} 


\section{Definition 10. Other-Side Population Monotonicity for Marriage Markets}

A solution $\varphi$ is other-side population monotonic if the following holds. Let $(N, R)$ be a marriage market and assume that $\left(N^{\prime}, R^{\prime}\right), N^{\prime}=N \cup\{n\}$, is an extension of $(N, R)$ and the newcomer $n$ is a man [woman]. Then, for all $\mu^{\prime} \in \varphi\left(N^{\prime}, R^{\prime}\right)$ there exists $\mu \in \varphi(N, R)$ such that (**) for all women $w \in N, \mu^{\prime}(w) R_{w} \mu(w)$ [for all men $m \in N, \mu^{\prime}(m) R_{m} \mu(m)$ ].

By the strictness of preferences, $(* *)$ means that if the newcomer is a man [woman], then every woman [man] who is matched differently is strictly better off, i.e., for all women $w \in N$, either $\mu^{\prime}(w)=\mu(w)$ or $\mu^{\prime}(w) P_{w} \mu(w)$ [for all men $m \in N$, either $\mu^{\prime}(m)=\mu(m)$ or $\mu^{\prime}(m) P_{m}$ $\mu(m)]$. Hence, if a woman $w[\operatorname{man} m]$ is unmatched from her mate at $\mu$, then she [he] is better off. Without specifying whether the newcomer is a man or a woman, other-side population monotonicity implies that if $m, w \in N$ are not matched anymore at $\mu^{\prime}$, then at least one of them is better off (if the newcomer is a man, then woman $w$ is better off and if the newcomer is a woman, then man $m$ is better off). This latter requirement that if two incumbents were unmatched at $\mu^{\prime}$, then one of them benefits from the increase of resources by the newcomer and is better off, can be formulated as a new property, namely resource sensitivity. This property requires that the solution is sensitive to an increase in resources, which is a different requirement than the solidarity aspect that other-side population monotonicity reflects.

\section{Definition 11. Resource Sensitivity}

A solution $\varphi$ is resource sensitive if the following holds. Let $(N, R)$ be a roommate market and assume that $\left(N^{\prime}, R^{\prime}\right), N^{\prime}=N \cup\{n\}$, is an extension of $(N, R)$. Then, for all $\mu^{\prime} \in \varphi\left(N^{\prime}, R^{\prime}\right)$ there exists $\mu \in \varphi(N, R)$ such that for all $i, j \in N$ [possibly $i=j$ ] that were mates at $\mu$ at least one is better off, i.e., if $i, j \in N, \mu(i)=j$, and $\mu^{\prime}(i) \neq j$, then $\mu^{\prime}(i) P_{i} \mu(i)$ or $\mu^{\prime}(j) P_{j} \mu(j)$.

On the class of marriage markets, resource sensitivity is essentially a weaker property than other-side population monotonicity.

\section{Lemma 2. Other-Side Population Monotonicity $\Rightarrow$ Resource Sensitivity}

On the class of marriage markets, individual rationality and other-side population monotonicity imply resource sensitivity.

Proof. Let $\varphi$ be a solution that is individually rational and other-side population monotonic on the class of marriage markets. Let $(N, R)$ be a marriage market and assume that $\left(N^{\prime}, R^{\prime}\right)$, $N^{\prime}=N \cup\{n\}$, is an extension of $(N, R)$. Without loss of generality assume that $n$ is a man. By other-side population monotonicity, for all $\mu^{\prime} \in \varphi\left(N^{\prime}, R^{\prime}\right)$ there exists $\mu \in \varphi(N, R)$ such that for all women $w \in N$, either $\mu^{\prime}(w)=\mu(w)$ or $\mu^{\prime}(w) P_{w} \mu(w)$. Let $i, j \in N, \mu(i)=j$, and $\mu^{\prime}(i) \neq j$. If $i \neq j$, then the pair $\{i, j\}$ consists of one man and one woman. Without loss of generality assume that $i$ is the man and $j$ the woman. Thus, by other-side population monotonicity, $\mu^{\prime}(j) P_{j} \mu(j)$. If $i=j$, then by individual rationality, $\mu^{\prime}(i) P_{i} \mu(i) .{ }^{9}$ Hence, $\varphi$ is resource sensitive.

Solution $\bar{\varphi}$ (Example 1) also demonstrates that for marriage markets, resource sensitivity is indeed a weaker condition than other-side population monotonicity: solution $\bar{\varphi}$ is individually rational and resource sensitive (see Proposition 2), but it violates other-side population monotonicity (see Appendix A).

\footnotetext{
${ }^{9}$ If $i$ is a woman, this latter implication would also be implied by other-side population monotonicity. However, if $i$ is a man, this concluding argument cannot be made solely by using other-side population monotonicity.
} 
Proposition 2. On the class of solvable roommate markets, any stable solution satisfies competition and resource sensitivity. In particular, the core satisfies competition and resource sensitivity.

Proof. Let $\varphi$ be a stable solution. Let $(N, R)$ be a solvable roommate market and assume that $\left(N^{\prime}, R^{\prime}\right), N^{\prime}=N \cup\{n\}$, is an extension of $(N, R)$ that is solvable.

Competition Sensitivity: Assume that the $\varphi$ is not competition sensitive, i.e., there exist $\mu \in \varphi(N, R), \mu^{\prime} \in \varphi\left(N^{\prime}, R^{\prime}\right)$, and $i, j \in N$ such that $\mu^{\prime}(i)=j, \mu(i) \neq j, \mu^{\prime}(i) P_{i} \mu(i)$, and $\mu^{\prime}(j) P_{j} \mu(j)$. But then $\{i, j\}$ is a blocking pair for $\mu$; contradicting $\mu \in \varphi(N, R) \subseteq S(N, R)$.

Resource Sensitivity: Assume that the $\varphi$ is not resource sensitive, i.e., there exist $\mu \in \varphi(N, R)$, $\mu^{\prime} \in \varphi\left(N^{\prime}, R^{\prime}\right)$, and $i, j \in N$ such that $\mu(i)=j, \mu^{\prime}(i) \neq j, \mu(i) P_{i} \mu^{\prime}(i)$, and $\mu(j) P_{j} \mu^{\prime}(j)$. But then $\{i, j\}$ is a blocking pair for $\mu^{\prime}$; contradicting $\mu^{\prime} \in \varphi\left(N^{\prime}, R^{\prime}\right) \subseteq S\left(N^{\prime}, R^{\prime}\right)$.

\section{Remark 1. A Straightforward Extension of Population Monotonicity?}

One might also consider the following "straightforward" extension of the two population monotonicity properties from marriage to roommate markets.

A solution $\varphi$ is "own-side population monotonic $(\star)$ " if for all roommate markets $(N, R)$, all newcomers $n$ such that $\left(N^{\prime}, R^{\prime}\right)$ is the roommate market obtained, all agents in $N$ that find the newcomer $n$ unacceptable weakly prefer $\varphi(N, R)$ to $\varphi\left(N^{\prime}, R^{\prime}\right)$.

A solution $\varphi$ is "other-side population monotonic $(\star)$ " if for all roommate markets $(N, R)$, all newcomers $n$ such that $\left(N^{\prime}, R^{\prime}\right)$ is the roommate market obtained, all agents in $N$ that find the newcomer $n$ acceptable weakly prefer $\varphi\left(N^{\prime}, R^{\prime}\right)$ to $\varphi(N, R)$.

The problem with this simple generalization of population monotonicity is that it is not clear which interesting solutions satisfy it: in Appendix B we demonstrate that no solution satisfying Pareto optimality and mutually best satisfies the straightforward population monotonicity extension discussed here.

\section{Results}

The following preference transformations will be used frequently in subsequent proofs.

Let $i, j \in N$ [possibly $i=j$ ] and $R_{i} \in L(N)$. Then, $R_{i}^{j} \in L(N)$ is obtained from $R_{i}$ by making $j$ the best match without changing the order over agents in $N \backslash\{j\}$, i.e., $R_{i}^{j}$ is such that for all $k \in N, j R_{i}^{j} k$ and for all $l, m \in N \backslash\{j\}, l R_{i}^{j} m$ if and only if $l R_{i} m$.

Let $i \in N, j \notin N, N^{\prime}=N \cup\{j\}$, and $R_{i} \in L(N)$. Then, $R_{i}^{j} \in L\left(N^{\prime}\right)$ is obtained from $R_{i}$ by making the newcomer $j$ the best match without changing the order over agents in $N$, i.e., $R_{i}^{j}$ is such that for all $k \in N, j P_{i}^{j} k$ and for all $l, m \in N, l R_{i}^{j} m$ if and only if $l R_{i} m$.

\subsection{Relations between Properties}

\section{Lemma 3.}

(a) On the class of solvable roommate markets, unanimity, Maskin monotonicity, and competition sensitivity imply mutually best.

(b) On the class of all roommate markets, unanimity, Maskin monotonicity, and competition sensitivity imply mutually best. 
Lemma 3 holds without using Maskin monotonicity. However, the proof without Maskin monotonicity is more involved (the problem without Maskin monotonicity occurs in the proof of Lemma 3 (a) where in each step the solvability of the constructed roommate market has to be guaranteed). Here we present the proof of Lemma 3 using Maskin monotonicity. In Appendix $\mathrm{C}$ we prove a stronger version of Lemma 3 using only weak unanimity and competition sensitivity. There, we also illustrate the complication that occurs without Maskin monotonicity in the following proof by means of an example.

Proof. Assume that $\varphi$ satisfies unanimity, Maskin monotonicity, and competition sensitivity, but not mutually best. Thus, there exists a (solvable) roommate market $(N, R)$ and a matching $\mu \in \varphi(N, R)$ such that for two agents $i$ and $j$ that are mutually best, $\mu(i) \neq j$.

We define $\tilde{R} \in \mathcal{R}^{N}$ as follows. For any $k \in N \backslash\{i, j\}$ we define $\tilde{R}_{k} \in L(N)$ by moving $\mu(k)$ on top of agent $k$ 's preferences, i.e., for all $k \in N \backslash\{i, j\}, \tilde{R}_{k}=R_{k}^{\mu(k)}$. Let $\tilde{R}_{i}=R_{i}$ and $\tilde{R}_{j}=R_{j}$. Note that $(N, \tilde{R})$ is solvable ${ }^{10}$ and that $\tilde{R}$ is a Maskin monotonic transformation of $R$ at $\mu$. Hence, by Maskin monotonicity, $\mu \in \varphi(N, \tilde{R})$. Without loss of generality assume that $\mu(i) \in N \backslash\{i\}$ or $\mu(j) \in N \backslash\{j\}$ (otherwise set $(\hat{N}, \hat{R})=(N, \tilde{R})$ and move to the last paragraph of the proof).

If $\mu(i) \in N \backslash\{i\}$, consider the extension $(\bar{N}, \bar{R})$ of $(N, \tilde{R})$ that is obtained by adding a newcomer $\bar{k}$ such that $\mu(i)$ and $\bar{k}$ are mutually best and $\bar{k}$ is unacceptable for all other agents $l \in N \backslash\{\mu(i)\}$, i.e., $\bar{N}=N \cup\{\bar{k}\}$ and $\bar{R} \in \mathcal{R}^{\bar{N}}$ is such that $\bar{R}_{\mu(i)}=\tilde{R}_{\mu(i)}^{\bar{k}}$, for all $k \in \bar{N}$, $\mu(i) \bar{R}_{\bar{k}} k$, and for all $l \in N \backslash\{\mu(i)\}, l \bar{P}_{l} \bar{k}$. Note that $(\bar{N}, \bar{R})$ is solvable. ${ }^{11}$ By competition sensitivity, for $\mu \in \varphi(N, \tilde{R})$, there exists $\bar{\mu} \in \varphi(\bar{N}, \bar{R})$ such that for all $i^{\prime}, j^{\prime} \in N$ [possibly $\left.i^{\prime}=j^{\prime}\right]$ that are new mates at $\bar{\mu}$ at least one is worse off. Hence, there exists $\bar{\mu} \in \varphi(\bar{N}, \bar{R})$ such that $\bar{\mu}(i) \neq j$. If $\mu(i)=i$ set $(\bar{N}, \bar{R})=(N, \tilde{R})$ and $\bar{\mu}=\mu$.

If $\mu(j) \in N \backslash\{j\}$, consider the extension $(\hat{N}, \hat{R})$ of $(\bar{N}, \bar{R})$ that is obtained by adding a newcomer $\hat{k}$ such that $\mu(j)$ and $\hat{k}$ are mutually best and $\hat{k}$ is unacceptable for all other agents $l \in \bar{N} \backslash\{\mu(j)\}$, i.e., $\hat{N}=\bar{N} \cup\{\hat{k}\}$ and $\hat{R} \in \mathcal{R}^{\hat{N}}$ is such that $\hat{R}_{\mu(j)}=\bar{R}_{\mu(j)}^{\hat{k}}$, for all $k \in \hat{N}$, $\mu(j) \hat{R}_{\hat{k}} k$, and for all $l \in \bar{N} \backslash\{\mu(j)\}, l \hat{P}_{l} \hat{k}$. Note that $(\hat{N}, \hat{R})$ is solvable. ${ }^{12}$ By competition sensitivity, for $\bar{\mu} \in \varphi(\bar{N}, \bar{R})$, there exists $\hat{\mu} \in \varphi(\hat{N}, \hat{R})$ such that for all $i^{\prime}, j^{\prime} \in N$ [possibly $\left.i^{\prime}=j^{\prime}\right]$ that are new mates at $\hat{\mu}$ at least one is worse off. Hence, there exists $\hat{\mu} \in \varphi(\hat{N}, \hat{R})$ such that $\hat{\mu}(i) \neq j$. If $\mu(j)=j$ set $(\hat{N}, \hat{R})=(\bar{N}, \bar{R})$ and $\hat{\mu}=\bar{\mu}$.

By construction, there now exists a unanimously best matching $\nu$ for $(\hat{N}, \hat{R}): \nu$ matches agent $i$ with agent $j$, agent $\mu(i)$ with agent $\bar{k}$ (if $\mu(i) \in N \backslash\{i\}$ ), agent $\mu(j)$ with agent $\hat{k}$ (if $\mu(j) \in N \backslash\{j\}$ ), and all agents in $\hat{N} \backslash\{i, j, \mu(i), \bar{k}, \mu(j), \hat{k}\}$ according to $\mu$. Hence, by unanimity, $\varphi(\hat{N}, \hat{R})=\{\nu\}$, contradicting $\hat{\mu} \in \varphi(\hat{N}, \hat{R})$.

\footnotetext{
${ }^{10}$ Roommate market $(N, \tilde{R})$ has a unique core allocation that matches agent $i$ with agent $j$ and all agents in $N \backslash\{i, j, \mu(i), \mu(j)\}$ according to $\mu$ - agent(s) $\mu(i)$ and $\mu(j)$ are either single or, if mutually acceptable, matched with each other.

${ }^{11}$ Roommate market $(\bar{N}, \bar{R})$ has a unique core allocation that matches agent $i$ with agent $j$, agent $\mu(i)$ with agent $\bar{k}$, and all agents in $\bar{N} \backslash\{i, j, \mu(i), \bar{k}, \mu(j)\}$ according to $\mu$-agent $\mu(j)$ is single.

${ }^{12}$ Roommate market $(\hat{N}, \hat{R})$ has a unique core allocation that matches agent $i$ with agent $j$, agent $\mu(i)$ with agent $\bar{k}$, agent $\mu(j)$ with agent $\hat{k}$, and all agents in $\hat{N} \backslash\{i, j, \mu(i), \bar{k}, \mu(j), \hat{k}\}$ according to $\mu$.
} 


\section{Lemma 4.}

(a) On the class of solvable roommate markets, unanimity, Maskin monotonicity, and resource sensitivity imply mutually best.

(b) On the class of all roommate markets, unanimity, Maskin monotonicity, and resource sensitivity imply mutually best.

It is an open question whether Lemma 4 holds on the domain of solvable roommate markets without using Maskin monotonicity. We discuss the reason why the proof strategy used to strengthen Lemma 3 by dropping Maskin monotonicity will not work for Lemma 4 in Appendix C. Interestingly, this illustrates that competition and resource sensitivity are not as symmetric as our main results suggest. In Appendix $\mathrm{C}$ we also illustrate the complication that occurs without Maskin monotonicity in the following proof by means of an example.

Proof. Assume that $\varphi$ satisfies unanimity, Maskin monotonicity, and resource sensitivity, but not mutually best. Thus, there exists a (solvable) roommate market $(N, R)$ and a matching $\mu \in \varphi(N, R)$ such that agents $i$ and $j$ are mutually best and $\mu(i) \neq j$.

We define $\tilde{R} \in \mathcal{R}^{N}$ as follows. For any $k \in N \backslash\{i, j\}$ we define $\tilde{R}_{k} \in L(N)$ by moving $\mu(k)$ on top of agent $k$ 's preferences, i.e., for all $k \in N \backslash\{i, j\}, \tilde{R}_{k}=R_{k}^{\mu(k)}$. Let $\tilde{R}_{i}=R_{i}$ and $\tilde{R}_{j}=R_{j}$. Note that $(N, \tilde{R})$ is solvable ${ }^{10}$ and that $\tilde{R}$ is a Maskin monotonic transformation of $R$ at $\mu$. Hence, by Maskin monotonicity, $\mu \in \varphi(N, \tilde{R})$.

Let $\bar{N}=[N \backslash\{\mu(i), \mu(j)\}] \cup\{i, j\}$ and define $\bar{R} \in \mathcal{R}^{\bar{N}}$ as the reduction of $\tilde{R}$ to $\bar{N}$, i.e., $\bar{R}=\tilde{R}_{\mid \bar{N}}$. There exists a unanimously best matching $\bar{\nu}$ for $(\bar{N}, \bar{R}): \bar{\nu}$ matches agent $i$ with agent $j$ and all agents in $\bar{N} \backslash\{i, j\}$ according to $\mu$. Hence, by unanimity, $\varphi(\bar{N}, \bar{R})=\{\bar{\nu}\}$. Without loss of generality assume that $\mu(i) \in N \backslash\{i\}$ or $\mu(j) \in N \backslash\{j\}$ (otherwise $(\bar{N}, \bar{R})=$ $(N, \tilde{R})$ and $\varphi(\bar{N}, \bar{R})=\{\bar{\nu}\}$ contradicts $\mu \in \varphi(N, \tilde{R}))$.

If $\mu(i) \in N \backslash\{i\}$, consider the extension $(\hat{N}, \hat{R})$ of $(\bar{N}, \bar{R})$ such that $\hat{N}=\bar{N} \cup\{\mu(i)\}$ and $\hat{R} \in \mathcal{R}^{\hat{N}}$ is the reduction of $\tilde{R}$ to $\hat{N}$, i.e., $\hat{R}=\tilde{R}_{\mid \hat{N}}$. Note that $(\hat{N}, \hat{R})$ is solvable. ${ }^{13}$ By resource sensitivity, for all $\hat{\mu} \in \varphi(\hat{N}, \hat{R})$ there exists $\bar{\mu} \in \varphi(\bar{N}, \bar{R})$ such that for all $i^{\prime}, j^{\prime} \in \bar{N}$ [possibly $\left.i^{\prime}=j^{\prime}\right]$ that were mates at $\bar{\mu}$ at least one is better off. Since $\varphi(\bar{N}, \bar{R})=\{\bar{\nu}\}$ was unanimously best and no agent $k \in \bar{N}$ prefers the newcomer $\mu(i)$ to his match $\bar{\nu}(k)$, by resource sensitivity, $\varphi(\hat{N}, \hat{R})=\{\hat{\nu}\}$ where $\hat{\nu}$ is such that for all $k \in \bar{N}, \hat{\nu}(k)=\bar{\nu}(k)$ and $\hat{\nu}(\mu(i))=\mu(i)$.

If $\mu(j) \in N \backslash\{j\}$, consider the extension $(N, \tilde{R})$ of $(\hat{N}, \hat{R})$. Recall that $(N, \tilde{R})$ is solvable. By resource sensitivity, for all $\tilde{\mu} \in \varphi(N, \tilde{R})$ there exists $\hat{\mu} \in \varphi(\hat{N}, \hat{R})$ such that for all $i^{\prime}, j^{\prime} \in \hat{N}$ [possibly $i^{\prime}=j^{\prime}$ ] that were mates at $\hat{\mu}$ at least one is better off. Since agents $i$ and $j$ are mutually best and $\varphi(\hat{N}, \hat{R})=\{\hat{\nu}\}$ with $\hat{\nu}(i)=j$, by resource sensitivity, for all $\tilde{\mu} \in \varphi(N, \tilde{R})$, $\tilde{\mu}(i)=j$; contradicting $\mu \in \varphi(N, \tilde{R})$.

\section{Lemma 5.}

(a) On the class of solvable roommate markets, mutually best and Maskin monotonicity imply individual rationality.

(b) On the class of all roommate markets, mutually best and Maskin monotonicity imply individual rationality.

\footnotetext{
${ }^{13}$ Roommate market $(\hat{N}, \hat{R})$ has a unique core allocation that matches agent $i$ with agent $j$ and all agents in $\hat{N} \backslash\{i, j, \mu(i)\}$ according to $\mu$-agent $\mu(i)$ is single.
} 
Proof. Assume that $\varphi$ satisfies mutually best and Maskin monotonicity, but not individual rationality. Thus, there exists a (solvable) roommate market $(N, R)$, a matching $\mu \in \varphi(N, R)$, and an agent $i \in N$ such that $i P_{i} \mu(i)$.

We define $\tilde{R} \in \mathcal{R}^{N}$ by moving $i$ on top of agent $i$ 's preferences and, for any $j \neq i$, by moving $\mu(j)$ on top of agent $j$ 's preferences, i.e., $\tilde{R}_{i}=R_{i}^{i}$ and for all $j \in N \backslash\{i\}, \tilde{R}_{j}=R_{j}^{\mu(j)}$. Note that $(N, \tilde{R})$ is solvable ${ }^{14}$ and that $\tilde{R}$ is a Maskin monotonic transformation of $R$ at $\mu$. Hence, by Maskin monotonicity, $\mu \in \varphi(N, \tilde{R})$. Let $\tilde{\mu}$ be the matching obtained from $\mu$ by unmatching agents $i$ and $\mu(i)$. By mutually best, $\varphi(N, \tilde{R})=\{\tilde{\mu}\}$. Since $\tilde{\mu} \neq \mu$ this is a contradiction to $\mu \in \varphi(N, \tilde{R})$.

\subsection{Two Characterizations of the Core and two Impossibilities}

\section{Lemma 6.}

(a) On the class of solvable roommate markets, if a solution $\varphi$ is mutually best and Maskin monotonic, then it is a subsolution of the core, i.e., for all roommate markets $(N, R)$, $\varphi(N, R) \subseteq \operatorname{core}(N, R)$.

(b) On the class of all roommate markets, no solution $\varphi$ is mutually best and Maskin monotonic.

Proof. Assume that $\varphi$ satisfies mutually best and Maskin monotonicity. By Lemma 5, $\varphi$ satisfies individual rationality.

To prove (a), suppose that there exists a solvable roommate market $(N, R)$ such that $\varphi(N, R) \nsubseteq \operatorname{core}(N, R)$. To prove (b), let $(N, R)$ be an unsolvable roommate market. In both cases there exists a matching $\mu \in \varphi(N, R)$ with a blocking pair $\{i, j\}$ for $\mu$. By individual rationality, $i \neq j$.

We define $\tilde{R} \in \mathcal{R}^{N}$ by moving $j$ on top of agent $i$ 's preferences, by moving $i$ on top of agent $j$ 's preferences and, for any $k \in N \backslash\{i, j\}$, by moving $\mu(k)$ on top of agent $k$ 's preferences, i.e., $\tilde{R}_{i}=R_{i}^{j}, \tilde{R}_{j}=R_{j}^{i}$, and for all $k \in N \backslash\{i, j\}, \tilde{R}_{k}=R_{k}^{\mu(k)}$. Note that $(N, \tilde{R})$ is solvable 15 and that $\tilde{R}$ is a Maskin monotonic transformation of $R$ at $\mu$. Hence, by Maskin monotonicity, $\mu \in \varphi(N, \tilde{R})$. By mutually best, for all $\tilde{\mu} \in \varphi(N, \tilde{R}), \tilde{\mu}(i)=j$. Since $\mu(i) \neq j$ this is a contradiction to $\mu \in \varphi(N, \tilde{R})$.

For (a) this proves that $\varphi(N, R) \subseteq \operatorname{core}(N, R)$ and for (b) this proves that mutually best and Maskin monotonicity are not compatible on the general domain of roommate markets.

Lemma 7. On the class of solvable roommate markets, there exists no Maskin monotonic strict subsolution of the core.

Proof. Sönmez's (1996, Theorem 1) result (which also applies for roommate markets) states that if a rule $\varphi$ is Pareto optimal, individually rational, and Maskin monotonic, then it is a supersolution of the core, i.e., for all roommate markets $(N, R), \varphi(N, R) \supseteq \operatorname{core}(N, R)$. Thus, since any subsolution of the core satisfies Pareto optimality and individual rationality, there exists no Maskin monotonic strict subsolution of the core.

\footnotetext{
${ }^{14}$ Roommate market $(N, \tilde{R})$ has a unique core allocation that matches all agents in $N \backslash\{i, \mu(i)\}$ according to $\mu$-agents $i$ and $\mu(i)$ are single.

${ }^{15}$ Roommate market $(N, \tilde{R})$ has a unique core allocation that matches agent $i$ with agent $j$ and all agents in $N \backslash\{i, j, \mu(i), \mu(j)\}$ according to $\mu-\operatorname{agent}(\mathrm{s}) \mu(i)$ and $\mu(j)$ are either single or, if mutually acceptable, matched with each other.
} 


\section{Theorem 1. Two Characterizations of the Core}

On the class of solvable roommate markets,

(a) a solution $\varphi$ satisfies unanimity, Maskin monotonicity, and competition sensitivity if and only if it equals the core;

(b) a solution $\varphi$ satisfies unanimity, Maskin monotonicity, and resource sensitivity if and only if it equals the core.

Proof. We consider the class of solvable roommate markets.

By Propositions 1 and 2, the core satisfies all properties listed in the theorem.

(a) Let $\varphi$ satisfy unanimity, Maskin monotonicity, and competition sensitivity. Then, by Lemma 3 (a), $\varphi$ satisfies mutually best.

(b) Let $\varphi$ satisfy unanimity, Maskin monotonicity, and resource sensitivity. Then, by Lemma 4 (a), $\varphi$ satisfies mutually best.

Thus, $\varphi$ satisfies Maskin monotonicity and mutually best. Hence, by Lemma 6 (a), $\varphi$ is a subsolution of the core. Since on the class of solvable roommate markets no Maskin monotonic strict subsolution of the core exists (Lemma 7), it follows that $\varphi=$ core.

\section{Theorem 2. Two Impossibility Results}

On the class of all roommate markets,

(a) no solution $\varphi$ satisfies unanimity, Maskin monotonicity, and competition sensitivity;

(b) no solution $\varphi$ satisfies unanimity, Maskin monotonicity, and resource sensitivity.

Proof. We consider the class of all roommate markets.

(a) Let $\varphi$ satisfy unanimity, Maskin monotonicity, and competition sensitivity. Then, by Lemma 3 (b), $\varphi$ satisfies mutually best.

(b) Let $\varphi$ satisfy unanimity, Maskin monotonicity, and resource sensitivity. Then, by Lemma 4 (b), $\varphi$ satisfies mutually best.

Thus, $\varphi$ satisfies Maskin monotonicity and mutually best; contradicting Lemma 6 (b).

We next show the independence of properties in Theorem 1.

The solution $\tilde{\varphi}$ that always assigns the matching at which all agents are single satisfies Maskin monotonicity, competition and resource sensitivity, but not unanimity.

Any strict subsolution of the core satisfies unanimity, competition and resource sensitivity (Proposition 2), but not Maskin monotonicity (Lemma 7).

The Pareto solution $P O$ satisfies unanimity and Maskin monotonicity, but - as the following two examples demonstrate - neither competition nor resource sensitivity.

Example 2. The Pareto Solution is not Competition Sensitive

Consider the solvable roommate markets $(N, R)$ and $\left(N^{\prime}, R^{\prime}\right)$ such that

\begin{tabular}{l|l}
$N=\{1,2\}$ & $N^{\prime}=\{1,2,3\}$ \\
\hline$R_{1}: 1,2$ & $R_{1}^{\prime}: 1,2,3$ \\
$R_{2}: 1,2$ & $R_{2}^{\prime}: 3,1,2$ \\
& $R_{3}^{\prime}: 2,3,1$ \\
& \\
$\operatorname{core}(N, R)=\{\bar{\mu}\}$ & $\begin{array}{l}\operatorname{core}\left(N^{\prime}, R^{\prime}\right)=\left\{\mu^{\prime}\right\} \\
\bar{\mu}=(1,2)\end{array}$ \\
$\mu^{\prime}=(1,3,2)$
\end{tabular}


Let $\mu=(2,1)$. Then, $P O(N, R)=\{\mu, \bar{\mu}\}$ and $P O\left(N^{\prime}, R^{\prime}\right)=\left\{\mu^{\prime}\right\}$. Thus, for $\mu \in P O(N, R)$ there exists $\mu^{\prime} \in P O\left(N^{\prime}, R^{\prime}\right)$ such that agent 1 is newly self matched at $\mu^{\prime}$ and better off. Hence, $P O$ violates competition sensitivity.

\section{Example 3. The Pareto Solution is not Resource Sensitive}

Consider the solvable roommate markets $(N, R)$ and $\left(N^{\prime}, R^{\prime}\right)$ such that

\begin{tabular}{l|l}
$N=\{1\}$ & $N^{\prime}=\{1,2\}$ \\
\hline$R_{1}: 1$ & $R_{1}^{\prime}: 1,2$ \\
& $R_{2}^{\prime}: 1,2$ \\
& \\
$\operatorname{core}(N, R)=\{\mu\}$ & $\operatorname{core}\left(N^{\prime}, R^{\prime}\right)=\{\bar{\mu}\}$ \\
$\mu=(1)$ & $\bar{\mu}=(1,2)$
\end{tabular}

Let $\mu^{\prime}=(2,1)$. Then, $P O(N, R)=\{\mu\}$ and $P O\left(N^{\prime}, R^{\prime}\right)=\left\{\mu^{\prime}, \bar{\mu}\right\}$. Thus, for $\mu^{\prime} \in P O\left(N^{\prime}, R^{\prime}\right)$ there exists $\mu \in P O(N, R)$ such that agent 1 was self matched at $\mu$ and is worse off at $\mu^{\prime}$. Hence, $P O$ violates resource sensitivity.

Finally, we briefly discuss the relation between both sensitivity conditions. Proposition 2 implies that competition and resource sensitivity are equivalent under stability. The following two solutions demonstrate that competition and resource sensitivity are logically independent. Let $\hat{\varphi}$ be the solution that always assigns the matching where all mutually best agents are mates and everybody else is single.

The following solution $\varphi^{\mathrm{CS}}$ satisfies competition sensitivity, but not resource sensitivity. For all solvable roommate markets $(N, R)$,

$$
\varphi^{\mathrm{CS}}(N, R)= \begin{cases}S(N, R) & \text { if } 1 \notin N \\ \hat{\varphi}(N, R) & \text { if } 1 \in N .\end{cases}
$$

The following solution $\varphi^{\mathrm{RS}}$ satisfies resource sensitivity, but not competition sensitivity. For all solvable roommate markets $(N, R)$,

$$
\varphi^{\mathrm{RS}}(N, R)= \begin{cases}\hat{\varphi}(N, R) & \text { if } 1 \notin N \\ S(N, R) & \text { if } 1 \in N .\end{cases}
$$

\subsection{Marriage Market Results}

Because we can easily adjust our proofs for the class of marriage markets, ${ }^{16}$ our results imply one of Toda's results.

Corollary 1. (Toda, 2006, Theorem 3.1)

On the class of marriage markets, the core is the unique solution satisfying weak unanimity, own-side population monotonicity, and Maskin monotonicity.

Finally, our results imply a new characterization of the core for marriage markets.

\section{Corollary 2.}

On the class of marriage markets, the core is the unique solution satisfying weak unanimity, other-side population monotonicity, and Maskin monotonicity.

\footnotetext{
${ }^{16}$ One only has to carefully choose the gender of newcomers in proofs.
} 


\section{References}

Barberà, S., Bossert, W., and Pattanaik, P. (2004): "Ranking Sets of Objects." In S. Barberà, P. Hammond, and C. Seidl, editors, Handbook of Utility Theory, volume 2. Kluwer Academic Publishers, Dordrecht.

Demange, G. and Wooders, M., editors (2004): Group Formation in Economics; Networks, Clubs and Coalitions. Cambridge University Press, Cambridge.

Gale, D. and Shapley, L. S. (1962): "College Admissions and the Stability of Marriage." American Mathematical Monthly, 69: 9-15.

Maskin, E. (1999): "Nash Equilibrium and Welfare Optimality." Review of Economic Studies, 66: $23-38$.

Roth, A. E. and Sotomayor, M. A. O. (1990): Two-Sided Matching: A Study in GameTheoretic Modeling and Analysis. Cambridge University Press, Cambridge.

Sönmez, T. (1996): "Implementation in Generalized Matching Problems." Journal of Mathematical Economics, 26: 429-439.

Thomson, W. (1983): "The Fair Division of a Fixed Supply Among a Growing Population." Mathematics of Operations Research, 8: 319-326.

Thomson, W. (1995): "Population Monotonic Allocation Rules." In W. A. Barnett, H. Moulin, M. Salles, and N. Schofield, editors, Social Choice, Welfare and Ethics. Cambridge University Press.

Toda, M. (2006): "Monotonicity and Consistency in Matching Markets." International Journal of Game Theory, 34: 13-31. 


\section{Appendix}

\section{A Solution $\bar{\varphi}$ is not Population Monotonic}

\section{Definition 12. Solution $\bar{\varphi}$}

We denote the single-valued solution that assigns to any marriage market its man-optimal [woman-optimal] stable matching by $\varphi^{M}\left[\varphi^{W}\right]$. Then, for all marriage markets $(N, R)$,

$$
\bar{\varphi}(N, R)= \begin{cases}\varphi^{M}(N, R) & \text { if } \max N \text { is a man } \\ \varphi^{W}(N, R) & \text { if } \max N \text { is a woman }\end{cases}
$$

In order to guarantee that $\bar{\varphi} \neq \varphi^{W}\left[\varphi^{M}\right]$, we assume that there exist women $w_{1}, w_{2} \in \mathbb{N}$ and men $m_{1}, m_{2} \in \mathbb{N}$ such that $w_{1}<m_{1}$ and $m_{2}<w_{2}$ (otherwise we relabel the agents in order to define $\left.\bar{\varphi} \neq \varphi^{W}\left[\varphi^{M}\right]\right)$.

Proposition 3. On the class of marriage markets, solution $\bar{\varphi}$ is individually rational, competition and resource sensitive, but neither own-side nor other-side population monotonic.

Proof. Since $\bar{\varphi}$ is a stable solution, it satisfies individual rationality and both sensitivity conditions (Proposition 2). The following examples demonstrate that $\bar{\varphi}$ is neither own-side nor other-side population monotonic. Assume that agents 1,2 , and 5 are men and agents 3 and 4 are women and consider roommate markets $(N, R)$ and $\left(N^{\prime}, R^{\prime}\right)$ such that

\begin{tabular}{l|l}
$N=\{1,2,3,4\}$ & $N^{\prime}=\{1,2,3,4,5\}$ \\
\hline & \\
$R_{1}: 3,4,1,2$ & $R_{1}^{\prime}: 3,4,1,2,5$ \\
$R_{2}: 4,3,2,1$ & $R_{2}^{\prime}: 4,3,2,1,5$ \\
$R_{3}: 2,1,3,4$ & $R_{3}^{\prime}: 2,1,3,4,5$ \\
$R_{4}: 1,2,4,3$ & $R_{4}^{\prime}: 1,2,4,3,5$ \\
& $R_{5}^{\prime}: 5, \ldots$ \\
$\bar{\varphi}(N, R)=\{\bar{\mu}\}$ & $\bar{\varphi}\left(N^{\prime}, R^{\prime}\right)=\left\{\mu^{\prime}\right\}$ \\
$\bar{\mu}=(4,3,2,1)$ & $\mu^{\prime}=(3,4,1,2,5)$
\end{tabular}

At marriage market $(N, R)$ woman 4 determines that the woman-optimal stable matching is chosen at $\bar{\varphi}(N, R)$ and man 1 is matched to woman 4 - his second choice. At marriage market $\left(N^{\prime}, R^{\prime}\right)$, man 5 causes a switch to the man-optimal stable matching at $\bar{\varphi}\left(N^{\prime}, R^{\prime}\right)$ and man 1 is now matched to woman 3 - his first choice. This is a violation of own-side population monotonicity. On the other hand, at marriage market $(N, R)$ woman 3 is matched to man 2 - her first choice. At marriage market $\left(N^{\prime}, R^{\prime}\right)$, woman 3 is matched to man 1 - her second choice. This is a violation of other-side population monotonicity. 


\section{B Straightforward Extensions of Population Monotonicity for Roommate Markets}

\section{Definition 13. Own-Side Population Monotonicity $(\star)$}

A solution $\varphi$ is own-side population monotonic $(\star)$ if the following holds. Let $(N, R)$ be a roommate market and assume that $\left(N^{\prime}, R^{\prime}\right), N^{\prime}=N \cup\{n\}$, is an extension of $(N, R)$ and $U\left(N^{\prime}, R^{\prime}, n\right)$ denotes the agents in $N$ that find agent $n$ unacceptable. Then, for all $\mu \in \varphi(N, R)$ there exists $\mu^{\prime} \in \varphi\left(N^{\prime}, R^{\prime}\right)$ such that for all $i \in U\left(N^{\prime}, R^{\prime}, n\right), \mu(i) R_{i} \mu^{\prime}(i)$.

\section{Definition 14. Other-Side Population Monotonicity $(\star)$}

A solution $\varphi$ is other-side population monotonic $(\star)$ if the following holds. Let $(N, R)$ be a roommate market and assume that $\left(N^{\prime}, R^{\prime}\right), N^{\prime}=N \cup\{n\}$, is an extension of $(N, R)$ and $A\left(N^{\prime}, R^{\prime}, n\right)$ denotes the agents in $N$ that find agent $n$ acceptable. Then, for all $\mu^{\prime} \in \varphi\left(N^{\prime}, R^{\prime}\right)$ there exists $\mu \in \varphi(N, R)$ such that for all $i \in A\left(N^{\prime}, R^{\prime}, n\right), \mu^{\prime}(i) R_{i} \mu(i)$.

Proposition 4. On the class of all roommate markets, no solution $\varphi$ satisfies Pareto optimality, mutually best, and own-side population monotonicity $(\star)$ [other-side population monotonicity $(\star)$ ].

Proof. Let solution $\varphi$ satisfy Pareto optimality and mutually best. Consider roommate markets $(N, R)$ and $\left(N^{\prime}, R^{\prime}\right)$ such that

\begin{tabular}{l|l}
$N=\{1,2,3\}$ & $N^{\prime}=\{1,2,3,4\}$ \\
\hline & \\
$R_{1}: 2,1,3$ & $R_{1}^{\prime}: 2,1,3,4$ \\
$R_{2}: 3,1,2$ & $R_{2}^{\prime}: 4,3,1,2$ \\
$R_{3}: 2,3,1$ & $R_{3}^{\prime}: 4,2,3,1$ \\
& $R_{4}^{\prime}: 3,4, \ldots$
\end{tabular}

Since $\varphi$ satisfies mutually best, for all $\mu \in \varphi(N, R), \mu(2)=3$ and for all $\mu^{\prime} \in \varphi\left(N^{\prime}, R^{\prime}\right)$, $\mu^{\prime}(3)=4$. Then, for all $\mu \in \varphi(N, R), \mu(1) \neq 2$ and by Pareto optimality, $\mu^{\prime}(1)=2$. Hence, in contradiction to own-side population monotonicity $(\star)$, agent $1 \in U\left(N^{\prime}, R^{\prime}, 4\right)$ always prefers his mate at $\left(N^{\prime}, R^{\prime}\right)$ to that at $(N, R)$. On the other hand, in contradiction to other-side population monotonicity $(\star)$, agent $2 \in A\left(N^{\prime}, R^{\prime}, 4\right)$ always prefers his mate at $(N, R)$ to that at $\left(N^{\prime}, R^{\prime}\right)$. 


\section{Stronger Versions of Lemmas 3 and 4}

The following example illustrates the difficulty we would encounter if we drop Maskin monotonicity from the proof of Lemma 3.

Example 4. The following two roommate markets $(N, R)$ and $(\bar{N}, \bar{R})$ are possible in the proof of Lemma 3 if no Maskin monotonic transformation is applied in the beginning of the proof (we assume $\mu(i) \neq i$ and $\mu(j)=j$ ).

\begin{tabular}{l|l}
$N=\{i, \mu(i), j, 1,2,3\}$ & $\bar{N}=N \cup\{\bar{k}\}$ \\
\hline & \\
$R_{i}: j, i, \ldots$ & $\bar{R}_{i}: j, i, \bar{k}, \ldots$ \\
$R_{j}: i, j, \ldots$ & $\bar{R}_{j}: i, j, \bar{k}, \ldots$ \\
$R_{1}: \mu(i), 2,3,1, \ldots$ & $\bar{R}_{1}: \mu(i), 2,3,1, \bar{k}, \ldots$ \\
$R_{2}: 3,1,2, \ldots$ & $\bar{R}_{2}: 3,1,2, \bar{k}, \ldots$ \\
$R_{3}: 1,2,3, \ldots$ & $\bar{R}_{3}: 1,2,3, \bar{k}, \ldots$ \\
$R_{\mu(i)}: i, 1, \mu(i) \ldots$ & $\bar{R}_{\mu(i)}: \bar{k}, i, 1, \mu(i), \ldots$ \\
& $\bar{R}_{\bar{k}}: \mu(i), \bar{k}, \ldots$
\end{tabular}

Note that the roommate market $(N, R)$ is solvable and has a unique core allocation that matches agent $i$ with agent $j$, agent $\mu(i)$ with agent 1 , and agent 2 with agent 3 . However, the roommate market $(\bar{N}, \bar{R})$ that resulted by adding newcomer $\bar{k}$ as in the proof of Lemma 3 is not solvable: at any stable matching agent $i$ should be matched with agent $j$ and agent $\mu(i)$ should be matched with agent $\bar{k}$. However, then there exists a "roommate cycle" for the remaining agents 1,2 , and 3 - they can never be matched in a stable way.

Next, we illustrate the difficulty we would encounter if we drop Maskin monotonicity from the proof of Lemma 4.

Example 5. The following two roommate markets $(N, R)$ and $(\bar{N}, \bar{R})$ are possible in the proof of Lemma 4 if no Maskin monotonic transformation is applied in the beginning of the proof (we assume $\mu(i) \neq i$ and $\mu(j)=j$ ).

\begin{tabular}{l|l}
$N=\{i, \mu(i), j, 1,2,3\}$ & $\bar{N}=N \backslash\{\mu(i)\}$ \\
\hline & \\
$R_{i}: j, i, \ldots$ & $\bar{R}_{i}: j, i, \ldots$ \\
$R_{j}: i, j, \ldots$ & $\bar{R}_{j}: i, j, \ldots$ \\
$R_{1}: \mu(i), 2,3,1, \ldots$ & $\bar{R}_{1}: 2,3,1, \ldots$ \\
$R_{2}: 3,1,2, \ldots$ & $\bar{R}_{2}: 3,1,2, \ldots$ \\
$R_{3}: 1,2,3, \ldots$ & $\bar{R}_{3}: 1,2,3, \ldots$ \\
$R_{\mu(i)}: i, 1, \mu(i) \ldots$ &
\end{tabular}

Note that the roommate market $(N, R)$ is solvable (see Example 4). However, the roommate market $(\bar{N}, \bar{R})$ that resulted by removing agents $\mu(i)$ and $\mu(j)$ as in the proof of Lemma 4 is not solvable: at any stable matching agent $i$ should be matched with agent $j$ and as in Example 4 we are left with a "roommate cycle" for the remaining agents 1, 2, and 3 - they can never be matched in a stable way. 
We will use the following weaker unanimity condition to strengthen Lemmas 3 and 4 .

\section{Definition 15. Weak Unanimity}

Let $(N, R)$ be a roommate market, $|N|$ even, and $\mu$ be a complete matching ${ }^{17}$ such that for all $i, j \in N, \mu(i) R_{i} j$. Then, $\mu$ is the unanimously best complete matching for $(N, R)$. A solution $\varphi$ is weakly unanimous if it chooses the unanimously best complete matching whenever it exists, i.e., for all roommate markets $(N, R)$ with an unanimously best complete matching $\mu$, $\varphi(N, R)=\{\mu\}$.

Next, we strengthen Lemma 3 in two ways. First, we replace unanimity by weak unanimity and second, we drop Maskin monotonicity.

\section{Lemma 3'.}

(a) On the class of solvable roommate markets, weak unanimity and competition sensitivity imply mutually best.

(b) On the class of all roommate markets, weak unanimity and competition sensitivity imply mutually best.

Proof. Assume that $\varphi$ satisfies weak unanimity and competition sensitivity, but not mutually best. Thus, there exists a (solvable) roommate market $(N, R)$ and a matching $\mu \in \varphi(N, R)$ such that for two agents $i$ and $j$ that are mutually best, $\mu(i) \neq j$.

First, if roommate market $(N, R)$ is solvable, we add newcomers in order to guarantee solvability in later steps. Thus, if $(N, R)$ is solvable then first go to Step 1 and otherwise go to Step 2 immediately.

Step 1: Guaranteeing Solvability Assume that roommate market $(N, R)$ is solvable and let $\mu^{\prime} \in S(N, R)$.

Let $k_{1}, k_{2}$ be such that $k_{1} \neq k_{2}$ and $\mu^{\prime}\left(k_{1}\right)=k_{2}$. Consider the extension $\left(N^{1}, R^{1}\right)$ of $(N, R)$ that is obtained by adding a newcomer $k_{1}^{\prime}$ such that $k_{2} P_{k_{1}} k_{1}^{\prime}$ (and for no $k \in N$, $\left.k_{2} P_{k_{1}} k P_{k_{1}} k_{1}^{\prime}\right)$ and for all $k \in N \backslash\left\{k_{1}\right\}, k_{1} P_{k_{1}^{\prime}} k_{1}^{\prime} P_{k_{1}^{\prime}} k$. Note that $\left(N^{1}, R^{1}\right)$ is solvable. ${ }^{18}$ By competition sensitivity, for $\mu \in \varphi(N, R)$, there exists $\mu^{1} \in \varphi\left(N^{1}, R^{1}\right)$ such that for all $i^{\prime}, j^{\prime} \in N$ [possibly $i^{\prime}=j^{\prime}$ ] that are new mates at $\mu^{1}$ at least one is worse off. Hence, there exists $\mu^{1} \in \varphi\left(N^{1}, R^{1}\right)$ such that $\mu^{1}(i) \neq j$ (agents $i$ and $j$ are still mutually best at $\left(N^{1}, R^{1}\right)$ ). Next, we consider the extension $\left(N^{2}, R^{2}\right)$ of $\left(N^{1}, R^{1}\right)$ that is obtained by adding a newcomer $k_{2}^{\prime}$ such that $k_{1} P_{k_{2}} k_{2}^{\prime}$ (and for no $k \in N, k_{1} P_{k_{2}} k P_{k_{2}} k_{2}^{\prime}$ ) and for all $k \in N \backslash\left\{k_{1}\right\}, k_{2} P_{k_{2}^{\prime}} k_{2}^{\prime} P_{k_{2}^{\prime}} k$. Similarly as before it follows that $\left(N^{2}, R^{2}\right)$ is solvable and there exists $\mu^{2} \in \varphi\left(N^{2}, R^{2}\right)$ such that $\mu^{2}(i) \neq j$ (agents $i$ and $j$ are still mutually best at $\left(N^{2}, R^{2}\right)$ ).

Note that we add newcomers as described above for all $k_{1}, k_{2}$ such that $k_{1} \neq k_{2}$ and $\mu^{\prime}\left(k_{1}\right)=k_{2}$. This results in a solvable roommate market that for notational convenience we also denote by $(N, R)$. For this matching market $(N, R)$ there exists a corresponding stable matching $\mu^{\prime}$ and a matching $\mu \in \varphi(N, R)$ such that for two mutually best agents $i$ and $j$, $\mu(i) \neq j$. The difference between this roommate market $(N, R)$ and the original market is that now a newcomer who is added in the sequel will not cause instability because an agent $k$ who is unmatched by the newcomer from his original stable partner at $\mu^{\prime}$ can now match in a stable way with the added agent $k^{\prime}$ instead of creating a "roommate cycle" (as in Example 4).

\footnotetext{
${ }^{17} \mathrm{~A}$ matching is complete if it partitions the set of agents into pairs, i.e., it contains no singletons.

${ }^{18}$ Roommate market $\left(N^{1}, R^{1}\right)$ has at least the stable matching where all agents in $N$ are matched according to $\mu^{\prime}$ and agent $k_{1}^{\prime}$ is single.
} 
Step 2: Without loss of generality assume that $N \backslash\{i, j\}=\{1,2, \ldots, l\}$. First, consider the extension $\left(N^{1}, R^{1}\right)$ of $(N, R)$ that is obtained by adding a newcomer $k_{1}$ such that agents 1 and $k_{1}$ are mutually best and $k_{1}$ is unacceptable for all other agents in $\{2, \ldots, l\}$. By competition sensitivity, for $\mu \in \varphi(N, R)$, there exists $\mu^{1} \in \varphi\left(N^{1}, R^{1}\right)$ such that for all $i^{\prime}, j^{\prime} \in N$ [possibly $\left.i^{\prime}=j^{\prime}\right]$ that are new mates at $\mu^{1}$ at least one is worse off. Hence, there exists $\mu^{1} \in \varphi\left(N^{1}, R^{1}\right)$ such that $\mu^{1}(i) \neq j$. Note that the pairs $\{i, j\}$ and $\left\{1, k_{1}\right\}$ consist of mutually best agents.

We continue adding newcomers $k_{2}, \ldots, k_{l}$ in a similar fashion and end up with a (solvable) roommate market $\left(N^{l}, R^{l}\right)$ such that there exists $\mu^{l} \in \varphi\left(N^{l}, R^{l}\right)$ with $\mu^{l}(i) \neq j$. Note that at $\left(N^{l}, R^{l}\right)$ we can partition $N^{l}$ in pairs $\{i, j\},\left\{1, k_{1}\right\}, \ldots,\left\{l, k_{l}\right\}$ of mutually best agents. Hence, a unanimously best complete matching $\nu$ for $\left(N^{l}, R^{l}\right)$ exists: $\nu$ matches agent $i$ with agent $j$, agent 1 with agent $k_{1}$, etc. Hence, by weak unanimity, $\varphi\left(N^{l}, R^{l}\right)=\{\nu\}$, contradicting $\mu^{l} \in \varphi\left(N^{l}, R^{l}\right)$.

Finally, we strengthen Lemma 4 by replacing unanimity by weak unanimity. We discuss the difficulties that occur when dropping Masking monotonicity after the proof.

\section{Lemma 4'.}

(a) On the class of solvable roommate markets, weak unanimity, Maskin monotonicity, and resource sensitivity imply mutually best.

(b) On the class of all roommate markets, weak unanimity, Maskin monotonicity, and resource sensitivity imply mutually best.

Proof. Assume that $\varphi$ satisfies weak unanimity, Maskin monotonicity, and resource sensitivity, but not mutually best. Thus, there exists a (solvable) roommate market $(N, R)$ and a matching $\mu \in \varphi(N, R)$ such that agents $i$ and $j$ are mutually best and $\mu(i) \neq j$.

We define $\tilde{R} \in \mathcal{R}^{N}$ as follows. For any $k \in N \backslash\{i, j\}$ we define $\tilde{R}_{k} \in L(N)$ by moving $\mu(k)$ on top of agent $k$ 's preferences, i.e., for all $k \in N \backslash\{i, j\}, \tilde{R}_{k}=R_{k}^{\mu(k)}$. Let $\tilde{R}_{i}=R_{i}$ and $\tilde{R}_{j}=R_{j}$. Note that $(N, \tilde{R})$ is solvable ${ }^{10}$ and that $\tilde{R}$ is a Maskin monotonic transformation of $R$ at $\mu$. Hence, by Maskin monotonicity, $\mu \in \varphi(N, \tilde{R})$, agents $i$ and $j$ are mutually best and $\mu(i) \neq j$.

Let $\bar{N}=\{i, j\}$ and consider the restriction $\bar{R}=\tilde{R}_{\bar{N}}$, i.e., $i \bar{R}_{j} j$ and $j \bar{R}_{i} i$ completely describes $\bar{R}$. There exists a unanimously best complete matching $\bar{\nu}$ for $(\bar{N}, \bar{R}): \bar{\nu}$ matches agent $i$ with agent $j$. Hence, by weak unanimity, $\varphi(\bar{N}, \bar{R})=\{\bar{\nu}\}$.

Without loss of generality assume that $N=\bar{N} \cup\{1, \ldots, l\}$. First, consider the extension $\left(N^{1}, R^{1}\right)$ of $(\bar{N}, \bar{R})$ that is obtained by adding newcomer 1 such that $N^{1}=\bar{N} \cup\{1\}$ and $R^{1}=\tilde{R}_{N^{1}}$. Note that $\left(N^{1}, R^{1}\right)$ is solvable. ${ }^{19}$ By resource sensitivity, for all $\mu^{1} \in \varphi\left(N^{1}, R^{1}\right)$, there exists $\bar{\mu} \in \varphi(\bar{N}, \bar{R})$ such that $\mu^{1}(i)=\bar{\mu}(i)=j$ or one of the agents $i$ and $j$ that were mates at $\bar{\mu}$ is better off. Since $\varphi(\bar{N}, \bar{R})=\{\bar{\nu}\}$ was unanimously best and neither agent $i$ nor agent $j$ prefer the newcomer to his match, by resource sensitivity, $\varphi\left(N^{1}, R^{1}\right)=\left\{\nu^{1}\right\}$ where $\nu^{1}$ is such that $\nu^{1}(i)=j$ and $\nu^{1}(1)=1$. Second, without loss of generality assume that $2=\mu(1)$ and consider the extension $\left(N^{2}, R^{2}\right)$ of $\left(N^{1}, R^{1}\right)$ that is obtained by adding newcomer $2 \mathrm{such}$ that $N^{2}=N^{1} \cup\{2\}$ and $R^{2}=\tilde{R}_{N^{2}}$. Note that $\left(N^{2}, R^{2}\right)$ is solvable. ${ }^{20}$ By resource sensitivity, for all $\mu^{2} \in \varphi\left(N^{2}, R^{2}\right)$ there exists $\mu^{1} \in \varphi\left(N^{1}, R^{1}\right)$ such that for all $i^{\prime}, j^{\prime} \in N^{1}$ [possibly $i^{\prime}=j^{\prime}$ ]

\footnotetext{
${ }^{19}$ Roommate market $\left(N^{1}, R^{1}\right)$ has a unique core allocation that matches agent $i$ with agent $j$ - agent 1 is single.

${ }^{20}$ Roommate market $\left(N^{2}, R^{2}\right)$ has a unique core allocation that matches agent $i$ with agent $j$ and agent 1 with agent 2 .
} 
that were mates at $\mu^{1}$ at least one is better off. Since agents $i$ and $j$ are mutually best and $\varphi\left(N^{1}, R^{1}\right)=\left\{\nu^{1}\right\}$ with $\nu^{1}(i)=j$, by resource sensitivity, for all $\mu^{2} \in \varphi\left(N^{2}, R^{2}\right), \mu^{2}(i)=j$.

We continue adding newcomers $3, \ldots, l$ in a similar fashion and end up with a solvable roommate market $\left(N^{l}, R^{l}\right)$ such that for all $\mu^{l} \in \varphi\left(N^{l}, R^{l}\right), \mu^{l}(i)=j$. Since $\left(N^{l}, R^{l}\right)=(N, \tilde{R})$, this contradicts $\mu \in \varphi(N, \tilde{R})$.

Finally we would like to comment why dropping Masking monotonicity is more difficult when resource sensitivity instead of competition sensitivity is used. Analyzing the proofs of Lemmas 3 and 4 we can - loosely speaking - say that competition sensitivity allows us to add agents to a roommate market and that resource sensitivity allows us to remove agents from a roommate market in order to obtain a roommate market for which unanimity narrows down the solution to a (weakly) unanimous matching. While it is always possible to create this unanimous solvable roommate market by adding extra agents (see Proof of Lemma 3') it might not be possible to create such a unanimous solvable roommate market by removing agents one at a time. The following example is a solvable roommate market from which we cannot remove any agent without destroying solvability.

Example 6. The following two roommate markets $(N, R)$ illustrates the difficulty of dropping Maskin monotonicity from Lemma 4.

$$
\begin{aligned}
& N=\{1,2,3,4\} \\
& R_{1}: 2,3,4,1 \\
& R_{2}: 3,4,1,2 \\
& R_{3}: 4,1,2,3 \\
& R_{4}: 1,2,3,4
\end{aligned}
$$

The unique stable matching for $(N, R)$ matches agents 1 and 3 and agents 2 and 4 . Removing any of the agents creates a "roommate cycle" for the remaining agents and the restricted roommate market is not solvable. 